\title{
Simulation of Refugee Flow in Europe Xiangyu Liu
}

\author{
School of North China Electric Power University Baoding, Baoding 071000, China
}

18730272577@163.com

Keywords: Refugees, AHP, min-cost max-flow algorithm, feed

\begin{abstract}
This paper focuses on four aspects: the factors affecting the movement of refugees, the optimal migration path, the allocation of resources and relevant policies to support our refugee model. Firstly, we adopt analytic hierarchy process(AHP) to analyze the factors and got a criterion to measure the refugee crisis. The analytic hierarchy process is consistent with the actual situation, which shows that the selected parameters are relatively reasonable. Secondly, we use the min-cost max-flow algorithm to develop a reasonable flow of refugees. Three strategies can be set correspondingly. The first strategy is regarded as the best, in which the number of casualties is minimum. Then multi-objective optimization dynamic programming model was used to realize the best allocation of resources in different regions. The result shows that the feed is the biggest consumption, followed by the capacity to house and protect. When the non-governmental organizations and other refugee destinations are added, the tendency of the change becomes gentle.
\end{abstract}

\section{Introduction}

Refugee crisis is one of the important issues facing the international community today. It not only affects the country that produces the refugee, but also has great influence on the region and even the whole world. Nowadays, with the development of globalization, the refugee problem is no longer a country, a region, but a global problem that the international community faces.

For example, Chowell et al. discussed the European refugee crisis begin in 2015, a rising number of refugees and migrants made the journey to the European Union to seek asylum, traveling across the Mediterranean Sea, or through Southeast Europe. They come from areas such as Western and South Africa, Africa, and the Western Balkans ${ }^{[1]}$. Althaus researched the structure of European refugees in the world's refugees, the structure of the source of refugees, the route to European from the angle of sociology of population, analyzed the causes of Europe produced refugee crisis and profound impact on the European region ${ }^{[2]}$.

\section{The Optimal Refugee Migration Model}

European refugee migration is a multi-objective risk strait, in the face of a huge number of complex factors. There are attributes of the individuals themselves, the routes they must take, the types of transportation, the countries' capacity, including number of entry points and resources available to refugee population.

\subsection{The establishment of Class hierarchy model}

Migration routes of refugees are generally divided into six routes, hence there exist six evaluation factors: the individuals themselves, the routes they must take, the types of transportation, the countries' capacity, the number of entry points and the available resources. Based on the optimal route evaluation factors and six routes, we can establish a hierarchical structure model as follows. 


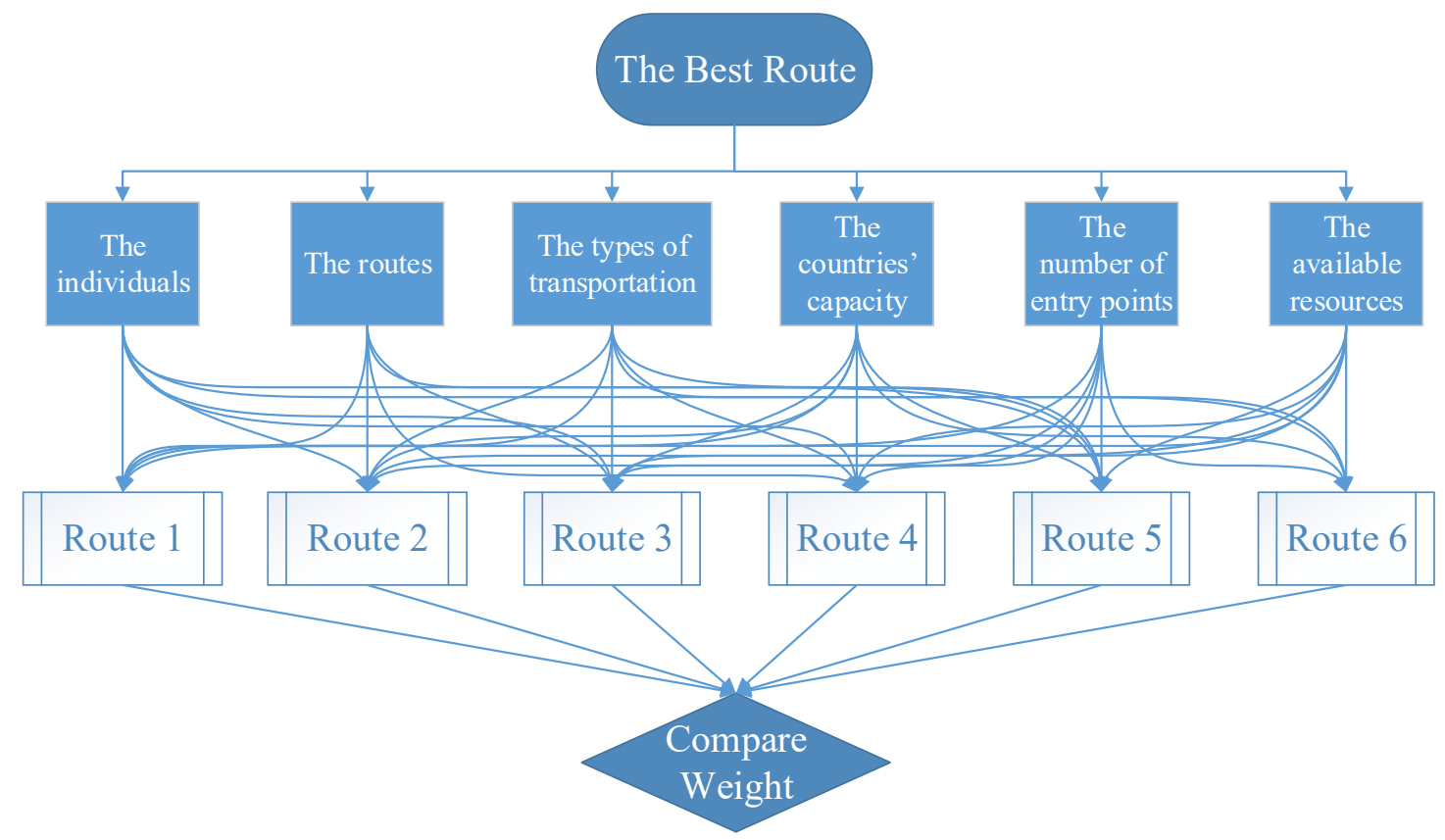

Figure1. The hierarchical structure model of the refugee crisis analysis

According to the safety and feasibility assessing the importance of each index, we can get the judged matrix $\boldsymbol{A}$. The value in each column approximately reflects the distribution of weights, using the arithmetic mean of all the column vectors to estimate weight vector $\mathbf{W}$ and $\lambda_{\max }$ :

$$
\boldsymbol{W}=\frac{1}{\boldsymbol{n}} \sum_{j=1}^{n} \frac{\boldsymbol{a}_{i j}}{\sum_{\boldsymbol{k}=1}^{\boldsymbol{n}} \boldsymbol{a}_{\boldsymbol{k} j}}, \quad \boldsymbol{i}=1,2, \cdots, \boldsymbol{n}
$$

$$
\lambda_{\max }=6.59
$$

Then, consistency test as follows:

$$
\begin{gathered}
\boldsymbol{C I}=\frac{\frac{\lambda_{\max }-n}{n-1}=\frac{6.59-6}{6-1}=0.1187}{C I} \\
\boldsymbol{C} \boldsymbol{R}=\frac{C I}{R I}=0.096<0.1, \text { where } \boldsymbol{R} \boldsymbol{I}=1.24
\end{gathered}
$$

The judged matrixes $\boldsymbol{B}_{\boldsymbol{i}}(\mathrm{i}=1,2, \ldots, 6)$ of the individuals themselves, the routes they must take, the types of transportation, the countries' capacity, the number of entry points and the available resources, the results of their consistency test. Calculating the $\boldsymbol{C R}_{\boldsymbol{i}}$ of $\boldsymbol{B}_{\boldsymbol{i}}(i=(1,2, \cdots, 6)), \boldsymbol{B}_{\boldsymbol{I}}$ surely pass the consistency test.

\subsection{The Establishment of Minimum cost Maximum flow Algorithm ${ }^{[3]}$}

Due to the different origin of refugees, we adopt the min-cost max-flow algorithm to arrange the route for refugees' migration reasonably. The departure of refugees and various of entry points in Europe as the vertexes, refugee migration routes as the directed arcs, a empowered communication directed graph $\boldsymbol{G}=(\boldsymbol{V}, \boldsymbol{E}, \boldsymbol{C})$ is established, where $\boldsymbol{V}$ is the set of vertices, $\boldsymbol{E}$ is the set of directed edges, $C$ is the capacity of the arc. First, we consider the origin of the refugees as $V s$, and it can be seen that $\boldsymbol{V} \boldsymbol{s}$ is not only. For simplicity, the areas of concentrated distribution of refugees are simplified several beginning points. In addition, the flows of refugee flow freely between the points, just restricted by geographical and martial factors. And then, with comprehensive assessment of such factors as the safety, cost and the number of entry points, $\boldsymbol{C}$ is calculated. At last, on account of the number and position of $\boldsymbol{V t}$ correspondingly changing, they need to be considered separately. We regard the European and the origin of refugees as the network apex, and use the minimum cost maximum flow algorithm to solve the flow of refugees. 


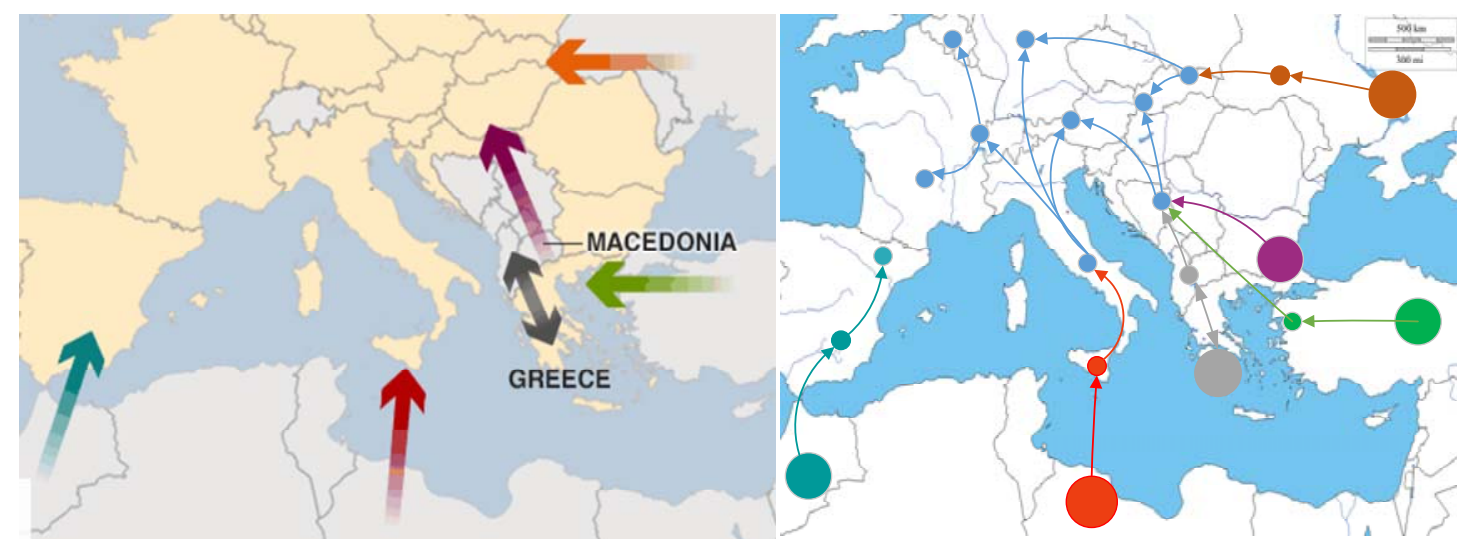

Figure 2. The Route of Flow of Refugees

\section{The Results of Minimum cost Maximum flow Algorithm}

From the above chart, using the arithmetic average method, we can get the weights of each scheme, so the total weight of Route4 is the largest.

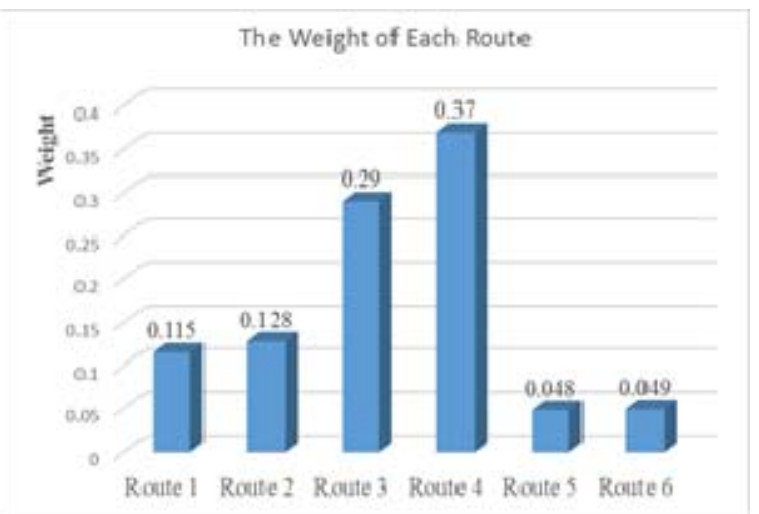

Figure 3. The weights of six routes

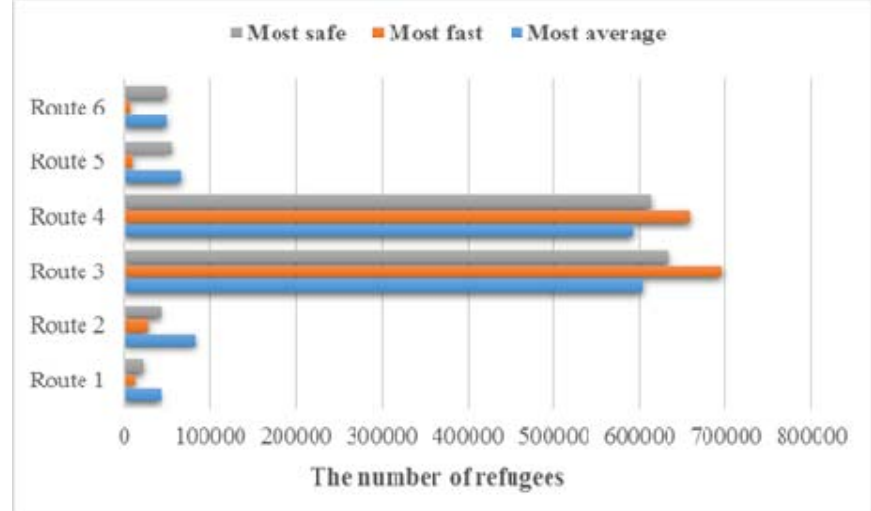

Figure 4. The number of refugees of each route

We can get the flow route of refugee by the minimum cost maximum flow algorithm. We can see that the Mediterranean route is still the most popular route, and the strategy $I$ is the best. Not only the number of refugee casualties reached the minimum, but the flow rate is also satisfactory.

There are some parameters that have a fixed value throughout our work. By varying the value of these parameters, we can see their impact on the model. The weight vector of $\boldsymbol{A}$ is modified as:

\begin{tabular}{lllllll}
\hline $\boldsymbol{\omega}_{1}$ & 0.04 & 0.25 & 0.05 & 0.39 & 0.08 & 0.20 \\
$\boldsymbol{\omega}_{2}$ & 0.08 & 0.20 & 0.09 & 0.30 & 0.11 & 0.22 \\
$\boldsymbol{\omega}_{3}$ & 0.12 & 0.15 & 0.13 & 0.21 & 0.14 & 0.24 \\
\hline
\end{tabular}

Table 1. The weight of A vector

We can be obtain the weight distribution of $\boldsymbol{B}_{\boldsymbol{i}}(\mathrm{i}=1,2, \ldots, 6)$ :

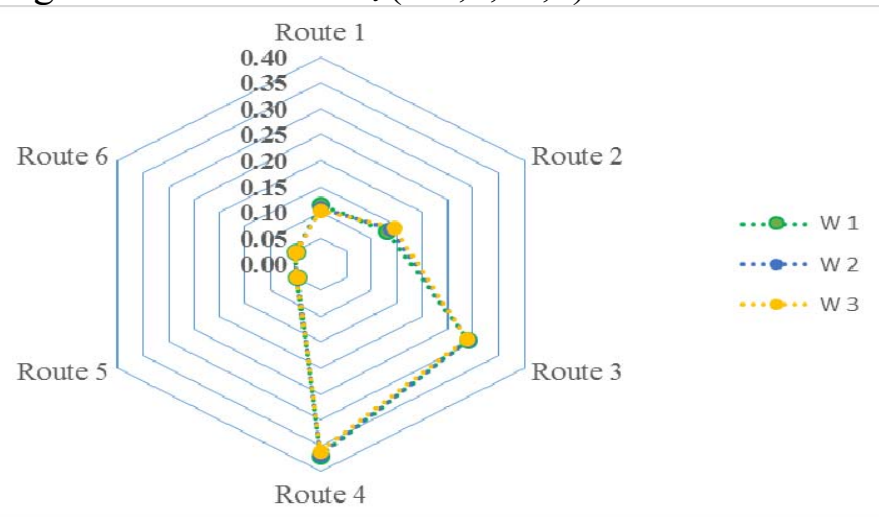

Figure 5. The result of Sensitivity Analysis

As it can be seen from the figure, although the weight vector of $\boldsymbol{A}$ constantly is changing, there are hardly differences among the results. From the side, Western Balkans and the Eastern Mediterranean, the two routes are better than other routes over various aspects. 


\section{Conclusions}

Multi-objective optimization dynamic programming model was used to realize the best allocation of resources in different regions. We studied the change of three resources, accommodation, health care and food. Our result shows that the feed is the biggest consumption, followed by the capacity to house and protect. Therefore, food needs priority. When the non-governmental organizations and other refugee destinations are added, the tendency of the change becomes gentle. Through the analysis of our refugee model, the policy should be setting as follows: European countries should expand the capacity of refugees and the war should be end as soon as possible.

We considered the impact of exogenous events, such as terrorist attack. The number of entry points and the routes they must take may be changed completely. With the change of the capacity of countries, the best migration strategy will change accordingly. Finally, we expand the crisis to a larger scale. According to our model, the speed of movement slows down obviously, and food problem gets more serious as well as health care. Thus, refugees must seek more asylums and the assistance of the international community was necessary.

\section{References}

[1] European migrant crisis. Wikipedia: https://en.wikipedia.org/wiki/European_migrant_crisis

[2] Althaus, Refugee crisis in Europe: structure, cause and effect analysis[J].

[3] Anja Wiesbrock(2011). The Integration of Immigrants in Sweden: a Model for the European Union [J]? INTERNATIONAL MIGRATION.

[4] Migrant crisis: Migration to Europe explained in graphics. BBC, 2015: http:// www.bbc.com /news/ world-europe-34131911

[5] International Organization for Migration. http://www.iom.int/

[6] UNHCR. http://www.unhcr.org/

[7] MIGRATION FLOWS - EUROPE. http://migration.iom.int/europe/ 